\title{
LA CRISIS DE LA SOCIEDAD CHILENA, NUEVA CONSTITUCIÓN Y PROCESO CONSTITUYENTE
}

\author{
Manuel Antonio Garretón Merino
}




\section{MANUEL ANTONIO GARRETÓN MERINO}

Premio Nacional de Humanidades y Ciencias Sociales 2007. Es sociólogo de la Pontificia Universidad Católica de Chile y Doctor de la Escuela de Altos Estudios en Ciencias Sociales, París. Es Profesor Titular de la Universidad de Chile. Ha sido Profesor invitado en instituciones tan prestigiosas como la Universidad de Oxford, Notre Dame, California en San Diego y Chicago, por nombrar algunas. Es autor de cerca de 60 libros entre autoría, co-autoría, ediciones y compilaciones, así como de alrededor de 350 artículos en revistas y libros, traducidos a varios idiomas. 


\section{LA CRISIS DE LA SOCIEDAD CHILENA, NUEVA CONSTITUCIÓN Y PROCESO CONSTITUYENTE*}

La idea central de este trabajo es que la sociedad chilena enfrenta una crisis de legitimidad del modelo de sociedad, de sus instituciones y de sus principales actores, y que una de las grandes oportunidades de salir de esta crisis es lo que se ha llamado el proceso constituyente que lleve a una nueva Constitución que reemplace la actual, heredada de la dictadura.

Luego de una cuestión conceptual sobre el tema constitucional, el presente trabajo analiza el sentido de una nueva Constitución en el caso chileno y la cuestión de la legitimidad de la Constitución actual a la luz de la crisis de las relaciones entre política y sociedad, lo que permitirá establecer la vinculación entre lo que se ha llamado los mecanismos y el contenido de una nueva Constitución. Se continúa señalando los contenidos posibles, enfatizando la dimensión de respuesta a los desafíos de la sociedad del siglo XXI para terminar con un análisis del actual proceso constituyente.

El enfoque de este artículo no es el jurídico constitucionalista ni el propio de especialistas constitucionales, sino de sociología política, perspectivas que no deben verse como contradictorias, sino como complementarias.

* Hemos tratado en particular muchos de los temas de este artículo en múltiples ocasiones. De modo que con el fin de no acudir repetidamente a citas, utilizamos directamente aquí algunos materiales expuestos en otros trabajos: "Informe de asesoría Legislativa. Análisis y comentario de proyecto de Nueva Constitución", Boletín No 10.193-07; "Plebiscito y Asamblea Constituyente para resolver el conflicto central de Chile", Revista Mensaje. No 642 Vol. LXIV, septiembre 2015; "El proceso constituyente y la disputa por su significado" (En Manuel Fernández y Cristian Figueroa eds. "Fumando opio' De la Asamblea Constituyente al poder ciudadano", Universidad Academia de Humanismo Cristiano, Mutante Editores, Santiago 2015); "Etapas y mecanismos del proceso constituyente" (En Claudio Fuentes y Alfredo Joignant, editores. "La solución constitucional. Plebiscitos, Asambleas, Congresos, sorteos y mecanismos híbridos", Catalonia, Santiago, 2015); "Proceso constituyente y relegitimación de la política. El papel de un plebiscito en la Nueva Constitución”" (En Revista Qué Pasa, 20 de octubre, 2015); "No se trata de desconfianza" (El Periodista, noviembre 2015); "El problema es la ruptura entre política y sociedad" (The Clinic, 17 de diciembre, 2015) 


\section{EL DOBLE SENTIDO DE UNA CONSTITUCIÓN Y EL PROBLEMA DE LEGITIMIDAD}

Desde una perspectiva socio-política, una Constitución expresa dos realidades (Aylwin y Pardo, 2015). Por un lado, es lo que constituye a una sociedad o a un Estado, es decir, un determinado orden socio-económico, cultural y político, una estructura real de poder de dicha sociedad. Por otro lado es un texto o ley constitucional que busca dar cuenta, y ello es siempre parcial, de lo que ese ordenamiento del poder en la sociedad es, pero también busca indicar y definir un tipo de ordenamiento o sociedad deseados, lo que se establece con la fórmula corriente: "la Constitución define el modo como queremos vivir como país".

En este doble sentido es que hay que evaluar la legitimidad de una Constitución. Tanto en cuanto si consagra un orden y relaciones de poder consideradas legítimas en una sociedad como en cuanto si su texto cumple con esas condiciones de legitimidad. Hay que entender este concepto en dos dimensiones: lo que se llama legitimidad de origen y legitimidad de ejercicio. Pero, a su vez, en esta última hay que distinguir entre legitimidad instrumental, que es la adhesión a algo porque permite usarlo o vivir en ello (depende de cual cosa estamos considerando en su legitimidad), satisfaciendo intereses de algunos (los que le dan legitimidad) o de todos, y legitimidad valórica, que consiste en la adhesión a algo porque se le considera lo bueno. Puede haber ilegitimidad en todas estas dimensiones o en algunas y, a su vez, cuando hay un conflicto entre diversos actores significativos entre quienes otorgan legitimidad y quienes la rechazan, hablamos no necesariamente de ilegitimidad, sino de crisis de legitimidad. (Habermas, 1973).

\section{LA CRISIS DE LEGITIMIDAD DE LA CONSTITUCIÓN DEL ‘80}

Desde todas estas perspectivas, la Constitución de 1980, impuesta por la dictadura y varias veces corregida, adolece de ilegitimidad o enfrenta una crisis de legitimidad.

En primer lugar, el orden socio-económico y la estructura de poder vigentes, con la excepción de los cambios en el régimen político, es fruto de la transformación operada por la dictadura sin ningún control democrático, y no ha podido ser sustituido por otro tanto por su propia capacidad reproductiva fáctica como por la normativa que lo respalda, entre otras, la propia Constitución. Los principios básicos de este orden son el predominio del mercado, el dinero, lo privado y particular por sobre lo colectivo, lo social, lo público y lo político, lo que tiene como consecuencia inevitable la desigualdad y el abuso de poder. (Garretón, 2012,2014). Su ilegitimidad es de origen, pues fue impuesto a través de la fuerza y coacción, 
violando y eliminando las normas precedentes, y valorativa, en la medida que no procede de un consenso o pacto social fundante ni prevé las posibilidades de cambio sustantivo, pero también instrumental, porque esa instrumentalidad es puramente adaptativa por parte de la mayoría de los actores de la sociedad y en los últimos años ha provocado múltiples reacciones de rechazo.

A su vez, el orden socio-político está también atravesado por una crisis de legitimidad no sólo de origen por las mismas razones que el orden socio-económico, sino también valórica, debido a la distancia y ruptura de la política con la sociedad, lo que quedó en evidencia con las movilizaciones de 2011-2012 y lo que se ha llamado crisis de confianza en las instituciones. Respecto de esto último, nuestra opinión es que es un error hablar de crisis de confianza como un problema de subjetividad: lo que hay, precisamente, es una ruptura de las formas clásicas de relación entre política y sociedad, sin un cambio por otras nuevas. A su vez, esta ruptura deslegitima desde la dimensión instrumental el orden político, como puede apreciarse en la baja de la participación electoral, que alcanzó menos del 50\% en la última elección presidencial.

En segundo lugar, el texto constitucional, que consagra un orden socioeconómico y político con crisis de legitimidad, tiene, a su vez, una ilegitimidad de origen. Si bien esto es indiscutible, lo que tiende a argumentarse es que todas las constituciones en Chile adolecieron de este déficit, pues no fueron hechas a través de mecanismos con participación ciudadana. Pero en este caso particular es el único en que ha habido un rechazo ciudadano posterior que convierte a este texto en ilegítimo desde el punto de vista valórico, desproveyéndolo de la legitimidad de ejercicio que teóricamente pueda haber tenido en un determinado momento.

En síntesis, el país carece de un consenso sobre su ordenamiento socioeconómico y político, lo que muchas veces se ha disfrazado bajo la idea de que hubo en los primeros gobiernos democráticos de la década del '90 una democracia de consensos y que la crisis actual se debería a la ausencia de tal política. En muchas ocasiones hemos insistido en que para el caso chileno de democratización, lo que se llamó democracia de los consensos no era tal, sino que hubo imposición de un orden por parte de quienes habían sido parte de la dictadura y una adaptación obligada o forzada de quienes se oponían, lo que corresponde de algún modo a la distinción de Rawls (1993) al referirse a 'overlapping consensus' (consensos básicos), lo que no existió en el caso chileno, y 'modus vivendi' (acuerdos de adaptación), que fue lo que realmente existió.

Todo ello puede resumirse en que, a diferencia de todos los países que han salido de dictaduras prolongadas, Chile no ha tenido su "momento constitucional" (Ackerman, 1999) y, por lo tanto, vive un orden constitucional en el doble sentido de 
orden socio-económico y político, y texto constitucional heredado de la dictadura y carente de legitimidad o en conflicto central de legitimidad.

\section{LA RUPTURA ENTRE INSTITUCIONALIDAD POLÍTICA Y SOCIEDAD}

Pero la crisis de legitimidad del orden socio-económico y político es sólo uno de los dos grandes problemas que atraviesa la sociedad chilena en su forma de estructurarse. Lo que Chile está experimentando es una ruptura en la relación clásica entre política institucional —incluidos los partidos, Congreso, Ejecutivo y otras instituciones - y sociedad, la que había consistido en la articulación o lo que hemos llamado imbricación entre el sistema partidario y los actores y movimientos sociales, a la vez imbricados entre sí, pero autónomos (Garretón, coord. 2016). Esta forma de acción social y política, propia de Chile desde la primera mitad del siglo $\mathrm{XX}$, permaneció en la época de la dictadura pese a la represión, y se expresó tanto en las movilizaciones contra el régimen que se desencadenan desde 1983 como en el plebiscito de 1988 que determinó el cambio de régimen. Ni la política logró asumir los cambios que experimentó la sociedad en democracia, ni el grueso de los actores sociales logró comprender la necesidad del momento e institucionalidad política. Ello, unido a un modelo económico social heredado de la dictadura, que fortalecía los poderes fácticos y mediáticos, y a la permanencia de un sistema político institucional que reproducía dicho modelo socioeconómico, pese a las correcciones en ambos aspectos debidas a los gobiernos de la Concertación, fue erosionando la articulación entre la sociedad y sus actores y los partidos e instituciones. Las movilizaciones de 2011-2012 y las elecciones presidenciales de 2013 dejaron de manifiesto esta ruptura, en el primer caso por la incapacidad de los actores políticos y sociales de entender que era necesario una nueva forma de articulación entre ellos; en el segundo caso porque si bien los programas recogían gran parte de las demandas de las movilizaciones, se alcanzaron porcentajes inéditos de baja participación. Los escándalos de corrupción posteriores confirmaron y agudizaron esta ruptura y la transformaron en crisis de confianza. La única solución posible en lo que será un largo proceso, si no se quiere acelerar esta descomposición del país como comunidad histórico política, es generar urgentemente hitos en que se produzca el reencuentro entre política y sociedad, para que se constituyan, en un marco institucional, nuevos actores y nuevas formas de articulación. 


\section{CONTENIDOS Y MECANISMOS DE UNA NUEVA CONSTITUCIÓN}

Una nueva Constitución, entonces, debe hacerse cargo de este doble problema: un nuevo texto constitucional que permita el cambio del actual ordenamiento socio-económico y político por uno que sea legítimo, pero al mismo tiempo, un modo de elaborarlo que se constituya en un hito para resolver la crisis estructural y orgánica entre política y sociedad o, si se quiere, la crisis de representación de la democracia actual. El momento constitucional está destinado a ser un proceso que busque resolver ambos problemas. Esto plantea una cuestión central que no siempre se considera en el debate: la relación indisoluble, en el caso chileno, entre el problema de fondo o los contenidos de la nueva Constitución, y el problema del método o mecanismo de elaboración ${ }^{1}$. De modo que es equivocada la posición de quienes sostienen que hay que discutir y acordar primero qué quiere cambiarse, los contenidos, y luego ponerse de acuerdo en los mecanismos. El error aquí es que o no se contesta la pregunta sobre quiénes discuten o entre quiénes es el acuerdo, o se da por supuesto que son los actuales actores políticos e institucionales (Congreso, partidos), precisamente aquellos cuya legitimidad está en crisis. Es evidente que es bueno generar instancias y espacios en que se discutan contenidos, por lo que no nos restamos en lo que sigue a plantear los que nos parecen fundamentales, aunque entendemos que es a través de un proceso constituyente que el pueblo o la ciudadanía deberá establecerlos.

\section{ELCONTEXTOEPOCALYNACIONALDEUNANUEVACONSTITUCIÓN}

Las nuevas constituciones, cuando inauguran o reemplazan las existentes, deben insertarse en dos contextos históricos. Uno, el que podría llamarse epocal en el nivel de la realidad mundial, y el otro en el nivel de la realidad de cada país ${ }^{2}$.

Respecto del contexto epocal, una Constitución que opta por los principios democráticos y republicanos de la organización socio-política en el marco de la doctrina de los derechos humanos, como es nuestro caso, debe asumir ciertas transformaciones y desafíos que cabe enunciar solamente, sin entrar a la discusión de su implementación en una nueva Constitución. Entre ellos: la crisis de las actuales formas de representación en los regímenes democráticos y la necesidad de

1. Sobre propuestas de nueva Constitución y mecanismos véase Fuentes y Joignant (2015), Fernández y Figueroa (2015), Garretón (2013), Varios autores (2013), Zúñiga (coord.) 2014.

2. Ver un desarrollo teórico y algunos análisis empíricos sobre el cambio constitucional en Negretto (2015). 
asegurar mecanismos de democracia directa y participativa, pero al mismo tiempo, la valoración de la democracia no sólo como régimen, sino como proceso y experiencia individual y colectiva; la expansión del concepto de ciudadanía alcanzando formas identitarias, pero que tiende a confundirse con una dimensión individualista asociada al consumo, lo que requiere reforzar el componente de responsabilidad que todo derecho acarrea, y la pertenencia a una comunidad política; la importancia de las respuestas regionales al proceso de globalización y de los fenómenos de democracia local; el debilitamiento de la pertenencia a la sociedad sobre la base de los principios de la nación concebida como una esencia ${ }^{3}$; la transformación y el cambio de sentido de instituciones tradicionales como la familia (cabe mejor hablar de "las familias") y de las organizaciones clásicas de convivencia; el papel de los medios de comunicación como poderes fácticos; la demanda de igualdad con sus múltiples dimensiones; la redefinición de las relaciones entre lo público y lo privado, en parte debido a las nuevas tecnologías de información y los cambios en la forma de convivencia ${ }^{4}$; el reconocimiento de los pueblos originarios, lo que obliga a establecer la plurinacionalidad del Estado y no sólo su pluriculturalidad.

Respecto del contexto histórico chileno, por un lado, los desafíos de la sociedad post industrial globalizada (Garretón, 2000, 2014), (que otros llaman sociedad del conocimiento, o de la información, o sociedad red, etc.), algunos de los cuales hemos indicado más arriba, y la doble tarea que se plantea a una nueva Constitución - nuevo orden socio-económico e institucional que supere al heredado de la dictadura y recomposición de las relaciones entre política y sociedad- definen una característica central que debe tener dicha Constitución. Ella no puede ser una Constitución mínima que sólo indique algunas reglas del juego y deje el resto a la acción de los poderes que la Constitución vigente impuesta ha consolidado. En la medida que se trata de refundar un orden socio-económico, reemplazando una Constitución que generó un orden socio-económico y político que ahora se intentaría superar, tendrá que ser una Constitución programática, congruente con lo que ha sido el "nuevo constitucionalismo latinoamericano" (Gargarella, 2015). Ello significa que junto con profundizar los derechos fundamentales y redefinir la relación entre poderes institucionales (sala de máquinas), lo que en las experiencias de cambio constitucional latinoamericano ha terminado por reforzar el presidencialismo, la Constitución no puede dejar de reflejar consensos básicos sobre un proyecto histórico de sociedad y Estado y sobre la real distribución de los poderes reales y no sólo institucionales. Esto supone también definirse en contrario

3. Habermas (2001) ha llamado al nuevo principio de pertenencia "patriotismo constitucional" opuesto al patriotismo tradicional

4. Sobre los cambios societales, Touraine (1997, 2013); Garretón (2000, 2014). Sobre las transformaciones de la democracia, Cheresky (2015); Rosanvallon (2007, 2015) 
a los principios del orden socio-económico y político heredado de la dictadura que se pretenden superar.

Recordemos que en el origen del debate sobre una nueva Constitución está la dictadura militar establecida en un golpe de Estado que destruyó la institucionalidad que se había dado el país. Ello hace necesario, ya sea en una definición programática, ya sea en el articulado, consagrar la condena de los actos violatorios de derechos cometidos por el Estado y el principio de nulidad de aquellas normas (ley de amnistía y otras), que estando vigentes contradigan los principios de la nueva Carta Fundamental, así como la no participación en cargos públicos de quienes hayan estado vinculados directa o indirectamente en la violación de derechos humanos.

Del mismo modo debe establecerse un horizonte del tipo de sociedad a que se aspira y en torno al cual se genere el patriotismo constitucional chileno, como lo han hecho las constituciones boliviana o ecuatoriana (PNUD, 2015), por ejemplo. No basta la consideración de un Estado de derechos, porque el basamento sigue individual y no de construcción de sociedad o polis. Esto implica un reforzamiento del principio de igualdad no concebido como equidad ni igualdad de oportunidades, sino igualdad socio-económica, política (poder) y cultural, sin perjuicio esta última del respeto y promoción de la diversidad cultural, entregando al Estado la responsabilidad de velar por asegurarla. En ese sentido cabe hablar de justa distribución del ingreso, de la riqueza y el poder y no sólo de ingresos. Del mismo modo cabe hablar de derecho a la propiedad y no sólo de propiedad. Todo ello significa que el Estado debe tener facultades de intervenir para evitar la concentración del poder económico, mediático.

\section{SOBRE LOS CONTENIDOS DE UNA NUEVA CONSTITUCIÓN}

Los objetivos clásicos de un texto constitucional son el establecimiento de las principios de organización de la sociedad y las bases de la soberanía, el establecimiento de los derechos fundamentales y la definición de la estructura de los poderes, lo que algunos llaman la "sala de máquinas" de la Constitución (Gargarella, 2015).

En conjunto, estos objetivos definen lo que el país quiere ser y cómo quiere establecer la convivencia entre sus miembros.

Respecto de los principios básicos que debieran informar una nueva Constitución, sin entrar en ellos de manera sistemática, indicaremos solamente algunas cuestiones que implican cambios sustantivos respecto de la actual Constitución ${ }^{5}$ :

5. Retomamos aquí los conceptos del Manifiesto Plebiscito para una Nueva Constitución, del que somos co-autores. En Varios Autores (2013). También Garretón (2013) 
Partamos de la afirmación de que el pueblo es el titular supremo y permanente de la soberanía, por ende, deben promoverse formas de expresión directa como el plebiscito, la iniciativa popular de ley, la posibilidad de revocatoria de mandatos y los organismos deliberativos y participativos en los diferentes niveles de decisión. Quienes gobiernan y deciden son las mayorías, con pleno respeto de los derechos fundamentales de las minorías. Esto supone un nuevo sistema electoral proporcional y la eliminación de los quórum calificados para la aprobación de ciertas leyes, como las llamadas orgánico-constitucionales, que tienen por efecto sobre-representar a las minorías y conferirles poder de veto sobre las decisiones mayoritarias. Del mismo modo, la instancia que se genere para resolver los problemas de constitucionalidad no puede tener facultades preventivas ni contra mayoritarias, lo que implica una radical modificación de las atribuciones y composición del actual Tribunal Constitucional. Asimismo, la definición actual de las Fuerzas Armadas debe ser profundamente revisada en el sentido de transformarlas en un cuerpo estrictamente profesional, no deliberante y dependiente del Ejecutivo, encargado sólo de tares de defensa externa y cooperación internacional, y en ningún caso constituir un poder del Estado con tareas de seguridad nacional, como se establece en la Constitución de 1980.

Por otro lado, las esferas de lo público y lo privado deben estar adecuadamente equilibradas en la regulación constitucional. Esto implica superar el carácter subsidiario del Estado en la economía y en la gestión de los intereses sociales por un rol dirigente y promotor en ámbitos como la educación, salud, previsión social, medios de comunicación y protección socio-ambiental. Asimismo, se requiere redimensionar el rol del derecho de propiedad, despojándolo del papel predominante y avasallador que tiene en la Constitución actual, y definir su función social, modificando el concepto por derecho a la propiedad. De esto se derivaría una nueva concepción del orden público económico y del marco regulador de las actividades empresariales del Estado y en el que todos los recursos naturales básicos tienen un carácter público, forman parte del patrimonio de la nación y deben ser resguardados y utilizados sustentablemente.

En materia de los derechos ciudadanos y los derechos de las diversas categorías e identidades sociales, especialmente las de género, infancia, juventud y adultos mayores y pueblos indígenas, deben ser adecuadamente protegidos, haciéndolos exigibles frente a los tribunales. En materia de derechos como son la educación y la libertad de expresión, el rol del Estado debe ser resaltado. Respecto del derecho a la educación, hay que considerar que ésta no es sólo un derecho de las personas, sino una función ineludible de toda sociedad y Estado, como lo es, por ejemplo, la

6. Retomamos aquí el planteamiento de F. Agüero, C, Fuentes y A. Varas (2016) 
defensa de la integridad territorial, que no puede reducirse a la suma de derechos individuales. En este sentido, es función del Estado garantizar su provisión en todos los niveles y gratuitamente, lo que resalta el papel predominante de la educación pública. En cuanto a la libertad de expresión, ella queda incompleta si no se asegura el papel del Estado en garantizar el pluralismo de los distintos tipos de medios de comunicación, ya sea creando sus propios medios públicos, ya sea subsidiando otros.

Respecto de la organización de los poderes, la arquitectura normativa de la nueva Constitución, en el marco de un Estado unitario, debe basarse en una nueva y más compensada fórmula de distribución del poder en la sociedad chilena, tanto entre gobernantes y gobernados, como entre los órganos del Estado entre sí, y entre el Gobierno central y los poderes regionales y locales. Ello significa, por un lado, la redefinición de un sistema de gobierno en términos de avanzar hacia un sistema semi-presidencial y, por otro, establecer modalidades más avanzadas de descentralización política y territorial, potenciando efectivamente a los gobiernos regionales elegidos para la conducción de sus regiones y abriendo la posibilidad de regímenes autonómicos para zonas del país habitadas mayoritariamente por pueblos originarios, cuyo reconocimiento constitucional es un deber histórico de justicia. El principio de participación que complemente al de representación en democracia debiera ser resaltado, generando el establecimiento de una estructura participativa en los diversos niveles de la sociedad y el Estado. En cuanto al voto, éste debe ser obligatorio, con sanciones altas y establecer la validez del voto blanco, de modo que en la ley consiguiente se establezca que superada una cierta proporción de votos blancos, la elección se repite.

\section{NUEVA CONSTITUCIÓN Y PROCESO CONSTITUYENTE}

Recordemos que la Presidenta Bachelet, en un acto histórico en nuestra vida republicana, anunció a mediados de 2015 un proceso constituyente que tenía un primer momento de tipo educativo, y un segundo momento participativo a través de encuentros locales y cabildos a lo largo del país, del que surgirían múltiples opiniones que serían recogidas por el Ejecutivo para elaborar un proyecto a presentarse ante la instancia que defina la nueva Constitución. En un tercer momento se enviará al Congreso actual un proyecto que le permita a éste, por dos tercios, delegar en el Congreso elegido en 2017, en un cuarto momento, la definición por tres quintos de sus miembros, del mecanismo o instancia para elaborar la nueva Constitución que pueden ser el Congreso mismo, una convención mixta de Congreso y ciudadanos, una asamblea constituyente o un plebiscito que decida entre las tres anteriores. 
Finalmente, un referéndum ratificará la Constitución propuesta por la instancia que se hubiera decidido.

Independientemente de los cambios que este itinerario pueda sufrir más adelante, cabe tomar en serio el desencadenamiento de un proceso constituyente.

Como hemos señalado, la necesidad que el país tiene de una nueva Constitución es tan imperiosa y urgente como la necesidad de restablecer o recomponer lazos entre ciudadanía y política, entre la sociedad y sus instituciones. De modo que cuando se piensa en mecanismos de elaboración de una nueva Constitución hay que pensar también en dotarlos de la legitimidad social, lo que sólo se consigue si se incorpora en ellos, en las diversas fases, la participación ciudadana. Un plebiscito para decidir el mecanismo, una asamblea constituyente para elaborar la nueva Constitución y un referéndum para ratificar el trabajo de la instancia constituyente, posibilitan mejor que cualquier otra fórmula el que política y sociedad vuelvan a encontrarse.

En virtud de asegurar la viabilidad, legitimidad y coherencia del proceso constituyente hay dos materias adicionales que deben discutirse. En primer lugar, los quórums indicados en la propuesta presidencial, por cuanto el requisito de dos tercios puede significar que el veto minoritario de la derecha, renuente a cualquier cambio, lleve a cancelar todo el proceso o lo condicione de tal manera que lo desnaturalice. En segundo lugar, debería establecerse el voto obligatorio para todas las instancias electorales del proceso.

\section{DESARROLLO Y PERSPECTIVAS DEL PROCESO}

Una vez anunciado el proceso constituyente, la Presidenta nominó un amplio Consejo de Observadores, con personas representativas de diversos sectores de la sociedad, con el fin de asegurar la transparencia y pluralismo del proceso. En realidad se trataba de dar garantías a los sectores de derecha y de la Democracia Cristiana que se habían mostrado renuentes al proceso mismo. La tarea de este Consejo duraría hasta el término de la etapa participativa, de la que daría cuenta en un informe final.

La primera etapa fue de tipo educativo y formativo y consistió en la elaboración de documentos, mensajes televisivos y otros elementos. La segunda etapa, de tipo participativo, siguiendo pautas elaboradas oficialmente y revisadas por el Consejo de Observadores (las que erróneamente no incluían el ítem del mecanismo) y con la participación de facilitadores o monitores, contempló primero Encuentros Locales Autoconvocados y Consultas Individuales en que se participaba por internet. El segundo momento fueron los Cabildos Provinciales y Regionales, que concluían el 7 de agosto. Todo el material será después procesado para ser considerado en 
el informe del Consejo de Observadores, pero sobre todo será la base para la elaboración del proyecto de nueva Constitución?

Desde el primer momento de anunciado el proceso se suscitó un fuerte debate por parte de la derecha, sus poderes mediáticos y sus portavoces, que rechazaba la idea misma de nueva Constitución y todo el proceso consiguiente. Por otro lado sectores dentro del Gobierno, incluidos no sólo democratacristianos, sino también de los partidos de izquierda, manifestaban su escepticismo. Sin embargo, en la medida en que el proceso fue mostrando avances, incluso las asociaciones empresariales y sectores de la derecha política se plegaron a él.

Más allá de las críticas que puedan hacerse a las modalidades seguidas hasta ahora y la evaluación del número de participantes, cabe señalar que estamos ante un proceso inédito en la historia de Chile y original respecto de otras experiencias internacionales, lo que muestra la consecuencia de la Presidenta. Lo cierto es que la etapa más compleja comienza ahora, en la medida que nos acercamos a los momentos decisorios. Es aquí donde los poderes fácticos y las elites políticas pueden conspirar contra la viabilidad de un auténtico proceso constituyente y momento constitucional.

Desde nuestra perspectiva, el mecanismo de elaboración de la nueva Constitución debiera ser una asamblea constituyente. Pero como no se cuenta al día de hoy con un consenso sobre ello y hay quienes quieren encapsular en el Congreso esta tarea, lo que le restaría toda legitimidad al proceso, dado el radical distanciamiento actual entre instituciones y sociedad a que nos hemos referido a lo largo de este texto, la fórmula para resolver cual sea el mecanismo es un plebiscito. Porque sería toda la ciudadanía la que resuelva el mecanismo y no eventualmente un Congreso que se auto otorga el papel de poder constituyente exclusivo. Por lo demás, si en el plebiscito se acordara que fuera el Congreso a través de una comisión bicameral o de una convención mixta — dos de los cuatro mecanismos alternativos propuestos - el que elaborara la Constitución, ésta gozaría de una legitimidad popular que no tendría si es el Congreso el que decide entre tales mecanismos. Del mismo modo, nadie podría rechazar una asamblea constituyente si ha sido decidida por un plebiscito. El plebiscito es la fórmula que le daría legitimidad a cualquier mecanismo posterior, porque además, en la memoria colectiva del pueblo chileno, está el plebiscito de 1988 que terminó con la dictadura, lo que hace natural y comprensible que sea a través de esta vía que se resuelva respecto de fórmulas

7. En total se realizaron 9.206 Encuentros Locales con más de 100.000 participantes, 82.993 Consultas Individuales, 71 Cabildos Provinciales con 12.852 participantes y hasta el 6 de agosto habían participado alrededor de 10.000 personas en los Cabildos Regionales. 
más complejas (como comisiones, convenciones o asamblea constituyente) para terminar con la Constitución impuesta por dicha dictadura. Se trata, sin duda, no de un mecanismo más, sino de la única manera democrática de resolver sobre qué mecanismos queremos para la nueva Constitución. Al mismo tiempo sería capaz de desencadenar un proceso que busque la mejor forma de resolver no sólo la cuestión constitucional, sino la profunda crisis de legitimidad de las instituciones y la política misma.

Y por ello es que, a diferencia de la propuesta de la Presidenta, nos parece que la primera reforma que debiera aprobar el Parlamento es la de llamar a un plebiscito para decidir el mecanismo (comisión bicameral, convención mixta o asamblea constituyente) y no considerar aquél como uno de los cuatro entre los que el Congreso debe decidir. Sin embargo, la ventaja de haber incluido el plebiscito como uno de los mecanismos para decidir quién elabora la Constitución y exigir tres quintos para tal decisión es que, como es prácticamente imposible lograr ese quórum para una de cuatro opciones, se establecerán debates y negociaciones. En ellas aparecerá que la auto atribución del Congreso de la facultad constituyente, como lo quisiera el polo democrático conservador de la política chilena, es inviable, porque contaría con el rechazo ciudadano, como lo han mostrado las instancias participativas del proceso hasta ahora, y que la única fórmula razonable, porque permite que la ciudadanía distanciada de las instituciones participe en el proceso, es el plebiscito para decidir el mecanismo que permita que la nueva Constitución cumpla con la tarea de superar la doble crisis de legitimidad de la sociedad chilena: la referida al orden socio-económico y político heredado de las dictaduras y la referida a la ruptura de las relaciones entre política y sociedad. 


\section{REFERENCIAS}

Ackerman, Bruce (1999), La política del diálogo liberal, Gedisa, Barcelona.

Agüero, Felipe. Varas, Augusto Fuentes y A. Varas (2016), La Nueva Constitución y las Fuerzas Armadas. Paper presentado en taller FFAA y Constitución, INAP-U de Chile y UDP, Santiago, mayo.

Aylwin, Sebastián, Pardo, Diego (2015), El procedimiento se va convirtiendo lentamente en sentencia. En Cuadernos de Coyuntura Número 10, Edición Primavera-Octubre, Santiago.

Cheresky, Isidoro (2015), El nuevo rostro de la democracia. Fondo de Cultura Económica. Buenos Aires.

Gargarella, Roberto (2015), La "sala de máquinas" de las Constituciones latinoamericanas. Lo viejo y lo nuevo. Revista Nueva Sociedad, No. 258. Julioagosto, Buenos Aires.

Fernández, Manuel y Figueroa, Cristián (2015) eds., "Fumando opio". De la Asamblea Constituyente alpoder ciudadano. Universidad Academia de Humanismo Cristiano, Mutante Editores, Santiago.

Fuentes, Claudio y Joignant, Alfredo (2015) eds., La solución constitucional. Plebiscitos, asambleas, congresos, sorteos y mecanismos bibridos. Catalonia, Santiago.

Garretón, Manuel Antonio (2000), La sociedad en que vivi(re)mos. Introducción sociológica al cambio de siglo. Santiago: LOM Ediciones.

-- -- -- - - - - -- -- -- -- -- -- -- -- -- -- -- (2012), Neoliberalismo corregido y progresismo limitado. Los gobiernos de la Concertación en Chile (1990-2010), Santiago: CLACSOARCIS.

-- -- - - - - - - - - - - - - (2013) El nuevo esfuerzo. Plebiscito para una nueva Constitución. En Varios autores, Plebiscito para una Constitución. Editorial El Desconcierto, Santiago.

-- -- -- -- (2014), Las ciencias sociales en la trama de Chile. Estudios sobre transformaciones sociopoliticas. LOM Ediciones, Santiago.

-- -- -- -- -- -- -- -- coordinador (2016), La gran ruptura. Institucionalidad política y actores sociales en el Chile del siglo XXI. LOM Ediciones, Santiago.

Garretón Manuel Antonio y Garretón, Roberto (2010), "La democracia incompleta en Chile: la realidad tras los rankings internacionales". Revista de Ciencia Politica, vol. 30, número 1, Santiago. 
Habermas, Jurgen. (2001), La constelación postnacional: ensayos políticos, Paidós, Barcelona.

-- -- -- - - - - (1973), Problemas de la legitimación en el capitalismo tardío. Amorrortu Editores, Buenos Aires.

Negretto, Gabriel (2015), La política del cambio constitucional en América Latina. Buenos Aires, Fondo de Cultura Económica.

PNUD (2015), Mecanismos de cambio constitucional en el mundo. Análisis desde la experiencia comparada. PNUD, Santiago, Chile

Rawls, John (1993). Political Liberalism. Nueva York: Columbia University Press.

Rosanvallon, Pierre (2007), La contrademocracia. La política en la era de la desconfianza. Ediciones Manantial, Buenos Aires.

-- - - - - - - - (2015) El buen gobierno. Ediciones Manatial, Buenos Aires

Touraine, Alain (1997), ¿Podremos vivir juntos? Iguales y Diferentes. México: Fondo de Cultura Económica.

-- - - (2013), La fin des sociétés. París: Editions du Seuil.

Varios autores (2013), Plebiscito para una Constitución. Editorial El Desconcierto, Santiago.

Zuñiga, Francisco (coordinador), Nueva Constitución y Momento Constitucional. Visiones, Antecedentes y Debates. Editorial Legal Publishing Thomson Reuters, 2014. 